\title{
Paper Performance Enhancement of Coherent Optical OFDM System Using LMS Algorithm
}

\author{
Manisha Bharti \\ Department of ECE, National Institute of Technology (NIT), Delhi, India
}

https://doi.org/10.26636/jtit.2020.137919

\begin{abstract}
Instability of the local oscillator causes phase noise - a phenomenon that is a disadvantage and is considered to be a major obstacle in the functioning of coherent optical orthogonal frequency division multiplexing (CO-OFDM) systems. An attempt has been made in this paper to reduce the effects of common phase errors generated by phase noise. In this paper, a least mean square (LMS) based algorithm is proposed for estimation of phase noise. Using this proposed algorithm, the major problem of phase ambiguity caused by cycle slip is avoided and the bit error rate is greatly improved. Further, there is no requirement for modifying the frame structure of OFDM using this algorithm. A CO-OFDM system with the 8-PSK technique is used to implement the algorithm concerned. Furthermore, the algorithm, using the 8-PSK modulation technique, is analyzed and compared with the existing QPSK technique and with other algorithms. The investigations reveal that 8-PSK outperforms existing LMS algorithms using other techniques and significantly reduces the bit error rate.
\end{abstract}

Keywords-coherent optical-orthogonal frequency division multiplexing (CO-OFDM), common phase error, least mean square (LMS), phase noise.

\section{Introduction}

Orthogonal frequency division multiplexing (OFDM) is an emerging technique for communicating through transmission channels characterized by frequency selective fading. Frequency selective fading is very difficult to handle in conventional communication receivers, as the design of the receiver becomes complex. Channel bandwidth is utilized efficiently using these multiplexing techniques just by dividing the total channel capacity into several low bandwidth channels [1]. OFDM alleviates this problem by conversion of a complete channel with frequency selective fading into several narrowband channels with flat fading, instead of mitigating the entire frequency selective fading. OFDM is a modulation technique in which a stream with a high data rate is divided into several closely spaced narrow bandwidth orthogonal subcarriers that are modulated at a lower bit rate. Such an approach makes this technique immune to frequency selective fading [2]. A conventional modulation scheme, such as phase shift keying (PSK), is used to modulate each subcarrier. OFDM is standardized in communications [3] under broadband wireless access system, LTE (4G), Wi-max (IEEE 802.16e), HIPERLAN and IEEE 802.11a standards (WLAN) [4]. Comparison with transmission schemes that use a single carrier shows that OFDM is effective in eradicating intersymbol interference (ISI) produced by multipath fading of the channel. It also provides a high transmission data rate and high efficiency of the spectrum. Moreover, it offers flexibility for adding and dropping optical signals. Therefore, it is suitable for future wireless communications operating at high speeds. However, the main limiting factor in using the orthogonal frequency division multiplexing technique is its sensitivity to phase noise. Phase noise is generated in a coherent optical OFDM system at both transmitter and receiver ends. At the transmitter side, this noise is induced by lase. On the other hand, at the receiver side, phase noise is formed by the local oscillator that is an essential element of receiver needed to produce local frequencies and to offer synchronization. Therefore, in order for the system to work efficiently, phase noise should be eliminated, which is considered to be a major degradation factor in the performance of a CO-OFDM system.

In this paper, an effort has been made to tackle the errors produced by phase noise with the help of the proposed algorithm that uses the CO-OFDM system in conjunction with the phase shift keying mechanism (8-PSK).

The paper is organized in the following manner. A brief description of OFDM is given in Section 1, followed by an explanation of the basic concepts of CO-OFDM and phase noise given in Section 2. In Section 3, the proposed algorithm is presented and explained. Investigations results and simulations are illustrated in Section 4. Finally, conclusions are presented in Section 5.

\section{Analysis of CO-OFDM System with Phase Noise}

Although OFDM was standardized in wireless communications a long time ago, CO-OFDM was first presented by researchers in 2008 and is considered to be the latest model used in optical communication systems. The coherent op- 
tical OFDM (CO-OFDM) is a technique in which input data is modulated using light frequency, and demodulation is performed in a coherent mode [5]. Two techniques of coherent detection and OFDM are combined in COOFDM. This scheme has many advantages in comparison to traditional techniques, with high electrical and spectral efficiency being one of them. Moreover, this technique is resistant to optical dispersion and shows high sensitivity at the receiver end. However, the phase noise produced by laser is a major drawback in the performance of the CO-OFDM system and must be compensated for. An efficient compensation mechanism is required in OFDM systems due to their long symbol sequences. Phase noise is generated in this system by laser used at the transmitter end and by the local oscillator used at the receiver end. Nonlinearity of optical fiber is a major source of phase noise as well [6]. The transmitter and the receiver of an OFDM system with phase noise are shown in Figs. 1 and 2, respectively. The OFDM scheme is based on dividing the high data rate stream into many lower data rate streams that can be transferred to orthogonal subcarriers. Each subcarrier is modulated using any traditional modulation scheme, such as the phase shift keying (PSK) technique or the quadrature amplitude modulation (QAM) scheme.

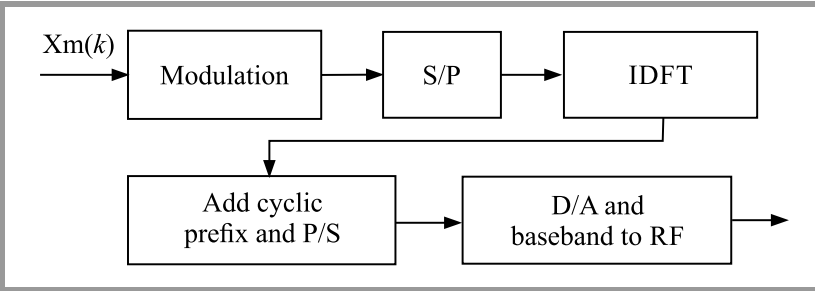

Fig. 1. Transmitter of an OFDM system.

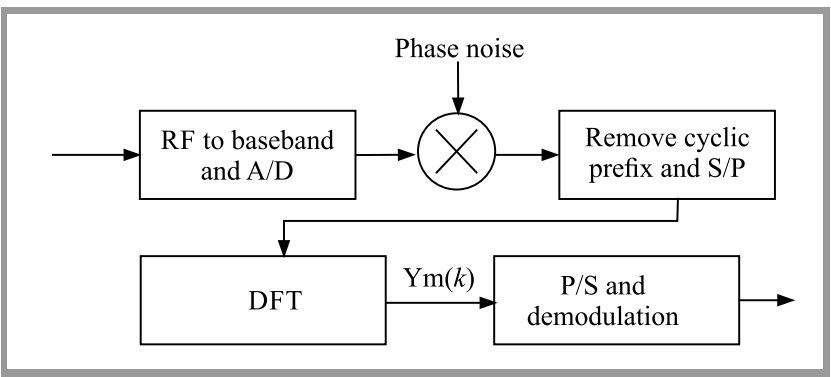

Fig. 2. Receiver of an OFDM system including phase noise.

At the transmitter, the OFDM system uses the source symbol in the frequency domain. These source symbols are used as inputs to IDFT block that converts the frequency domain into time domain. OFDM converts the incoming symbols into $N$ signals of a lower rate. These signals modulate the subcarriers that are orthogonal to each other, using IDFT. The time-domain signals are obtained from IDFT [7]. The carrier signal obtained is represented as:

$$
c(t)=A_{c}(t) \mathrm{e}^{\mathrm{j}\left|w_{s} t+\emptyset_{c} t\right|},
$$

where $A_{c}(t)$ denotes the amplitude of the carrier.
Complex signal $C_{S}(t)$ is given by:

$$
c_{S}(t)=\frac{1}{N} \sum_{n=0}^{n=N-1} A_{t}(t) \mathrm{e}^{\mathrm{j}\left[w_{n} t+\varnothing_{n} t\right]} .
$$

After sampling the above signal, the signal is given by:

$$
S_{S}(k t)=\frac{1}{N} \sum_{n=0}^{n=N-1} A_{n} \mathrm{e}^{\mathrm{j}\left[\left(w_{0} t+n \Delta w\right) k t+\emptyset_{n}\right]} .
$$

The phase noise produced actually destroys the main feature of orthogonality among the subcarriers, which leads to a reduction in the system's performance [8]. Phase noise causes the symbols transmitted to be rotated by a small angle - the phenomenon is known as common phase error (CPE) [9]. Using a CPE-sensitive constellation, many errors result due to uncompensated CPE. Hence, there is a need to compensate for the phase error before detection of the symbols [10]-[12] at the receiver end.

An OFDM system having $\mathrm{N}$ subcarriers, and characterized by the symbol duration $\mathrm{T}$ and cyclic prefix length $N_{g}$ (to eliminate ISI caused by multipath fading) is considered in this paper. For $n$-th subcarrier of the $m$-th symbol phase, noise is:

$$
\begin{gathered}
\emptyset_{m}(n)=\emptyset_{m-1}(N-1)+\sum_{n=-N_{g}}^{n=N} u\left[m\left(N+N_{g}\right)+i\right], \\
\emptyset_{m}(n)=\sum_{i=0}^{i=m\left(N+N_{g}\right)+N_{g}+n} u(i) .
\end{gathered}
$$

Here, $u(i)$ denotes the mutually independent random variables having a Gaussian probability density function with a zero mean.

The correlation function value of $\emptyset_{m}(n)$ between the same symbol is given as:

$$
\begin{aligned}
E\left[\emptyset_{m}(n) \cdot \varnothing_{m}^{*}(I)\right]=\left(\frac{2 \pi B T}{N}\right)^{*} \min [ & {\left[N\left(N_{g}\right)\right.} \\
+ & \left.n, m\left(N+N_{g}\right)+I\right] .
\end{aligned}
$$

From Eqs. (4)-(6), it is implied that phase noise is basically a random process that is non-stationary, with a time varying correlation function. CPE causes rotation of the signal constellation by a specific angle, and is worked out as:

$$
\emptyset_{0}=\frac{1}{T_{s}} \int_{0}^{T_{s}} j(t) \cdot \mathrm{d} t
$$

\section{Proposed Algorithm}

Out of many phase noise estimation algorithms used in OFDM systems, the use of feedback-adaptive estimation based on LMS seems to be one of the best choices. In this estimation algorithm, phase-related information from the previous symbols is used to generate an estimate for the present symbol. Nevertheless, the bit error rate (BER) produced by this algorithm is rather high. It uses a modulation 
scheme of QPSK and has an ambiguity of $90^{\circ}$ in phase. If the instantaneous phase error is greater than $45^{\circ}$ due to the combined effects of line-widths and noise, the carrier will be recovered back from the lock point that is $90^{\circ}$ away from the phase of the current pulse, thereby inducing the error rate in coherent detection. This is the problem of cycle slip which is a major obstacle affecting performance of the system [14] when this specific technique is used.

Figure 3 shows a schematic representation of the proposed algorithm. In the first stage, pilot symbols are utilized to track the phase of the signal. The received sequence is then multiplied by the complex conjugate part of the pilot sequence, which is taken as zero-padded. Further interpolation filters are used to obtain the resultant sequence.

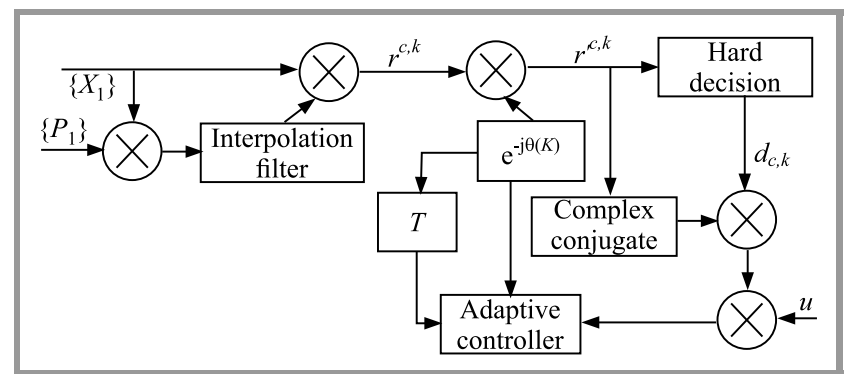

Fig. 3. Diagram of the proposed algorithm.

The argument of the samples is then taken for the phase correction at the output of the interpolation filter. The residual phase offset is then corrected by the adaptive LMS estimation algorithm, which does not suffer from ambiguity in phase [15]. This implies that only a small phase-offset is obtained from the sequence which is at the input of the second stage. The estimation of phase produced by it lies within the range of $-\pi / 4$ to $+\pi / 4$. Earlier, the system was designed by using an adaptive algorithm which was not able to demonstrate good performance due to a low SNR value. Performance of this system was degraded mainly by cycle-slips that were produced by the carrier frequency signal slipping to the next lock point location. Then, the CO-OFDM system was designed and demonstrated, using the QPSK technique. Using this technique, the problem of cycle slip was resolved by limiting the value of phase offset to a pre-decided range. The BER was not improved significantly, however. The proposed algorithm shows a remarkable difference in the value of SNR at the same BER. Furthermore, due to a limited rotation angle range, no cycle slip is observed in this algorithm. Moreover, this algorithm does not introduce any changes to the OFDM frame structure.

\section{Results and Discussions}

Matlab is used for generating the OFDM signal and for analyzing system performance. A 256-size IFFT/FFT is used, comprising of 1 pilot symbol and 255 OFDM subcarriers. 8-PSK and QPSK modulation techniques are used in simulating the algorithm. By using M-ary schemes, i.e. when a signal is represented to map by more than a single bit, bandwidth efficiency is achieved. A phase change of $180^{\circ}$ represents digital data of 1 and 0 in BPSK modulation. 2 bits are mapped on each signal in QPSK by a phase shift of $90^{\circ}$. By employing several phases in M-ary PSK, more than 2 bits may be mapped. In QPSK, data is encoded by utilizing four distinct shifts of phase, i.e. $45^{\circ}, 225^{\circ}, 135^{\circ}$, and $315^{\circ}$. However, in the 8-PSK, scheme, eight distinct phase-shifts, i.e. $0^{\circ}, 45^{\circ}, 90^{\circ}, 135^{\circ}, 180^{\circ}, 225^{\circ}, 270^{\circ}$ and $315^{\circ}$ are used for encoding the data. In 8-PSK modulation, the complete coordinate system is evenly divided into 8 angle sectors representing 8 constellation symbols. One symbol is formed by mapping 3 bits in 8-PSK modulation. Various algorithms that deal with the phase noise error are compared on the basis of BER and SNR. The techniques that are implemented and compared include adaptive LMS, theoretical QPSK, improved 2-stage and the proposed technique of LMS with 8-PSK. Performance metrics, such as constellation diagram, BER and SNR, are used to evaluate the system's performance.

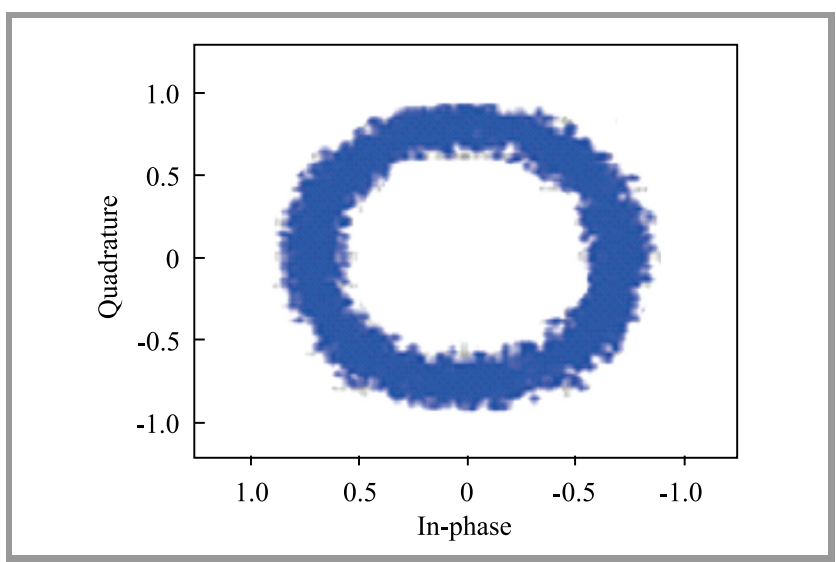

Fig. 4. Constellation points of 8-PSK before compensation.

Figure 4 shows the constellation diagram of 8-PSK before compensation of phase noise. It may be clearly seen in the figure that noise has fully contaminated the system, adversely affecting its performance.

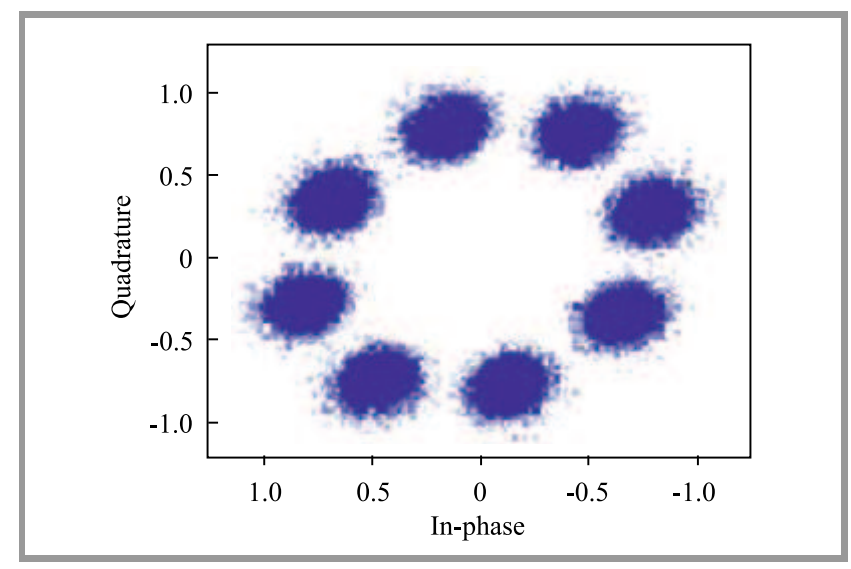

Fig. 5. Constellation points of 8-PSK after compensation in the first stage. 


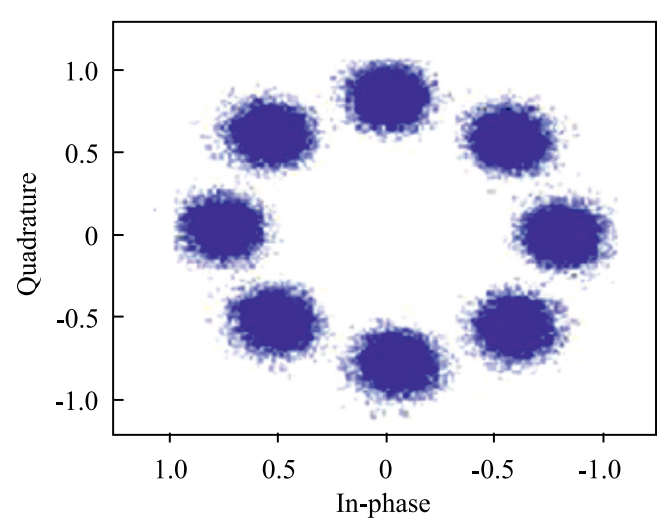

Fig. 6. Constellation points of 8-PSK after compensation in the second stage.

Figures 5-6 show the constellation points of 8-PSK after compensation of phase noise in the first and second stage, respectively. Pilot symbols are used to correct the constellation points in the first stage and then in the second stage. These constellation points are corrected and phase noise is compensated using the LMS phase estimation algorithm.

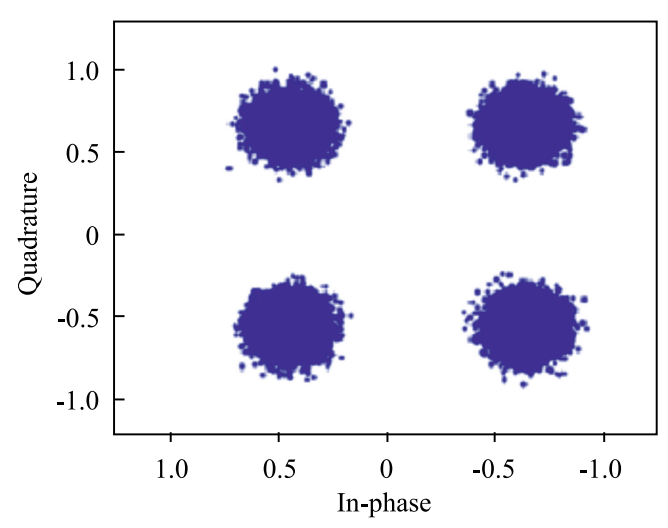

Fig. 7. Constellation points of QPSK after compensation in the first stage.

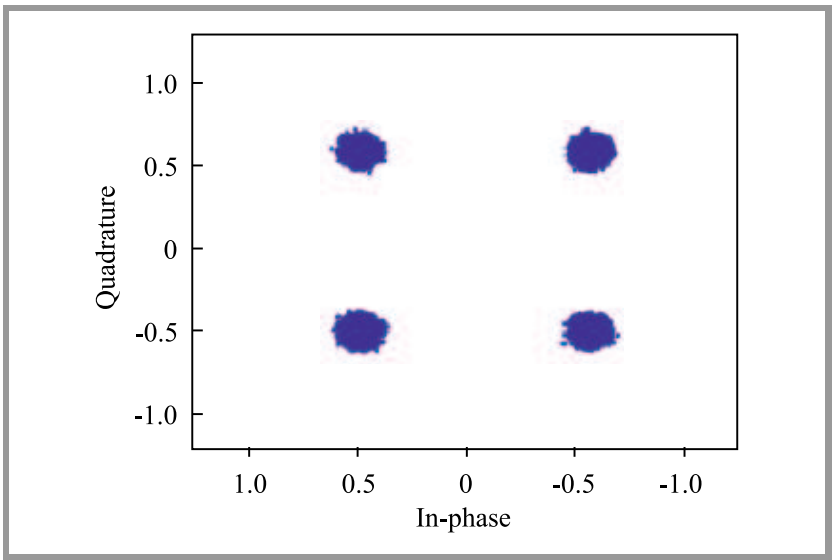

Fig. 8. Constellation points of QPSK after compensation in the second stage.
The QPSK constellation points after phase noise compensation performed in the first stage using pilot symbols and in the second stage using the LMS-based algorithm, are depicted in Figs. 7-8.

Finally, Fig. 9 demonstrates a comparative analysis of the bit error rate, between the algorithms proposed, using 8-PSK modulation scheme and the existing algorithms using QPSK and other approaches. Performance of the adaptive LMS algorithm, the improved two stage algorithm with

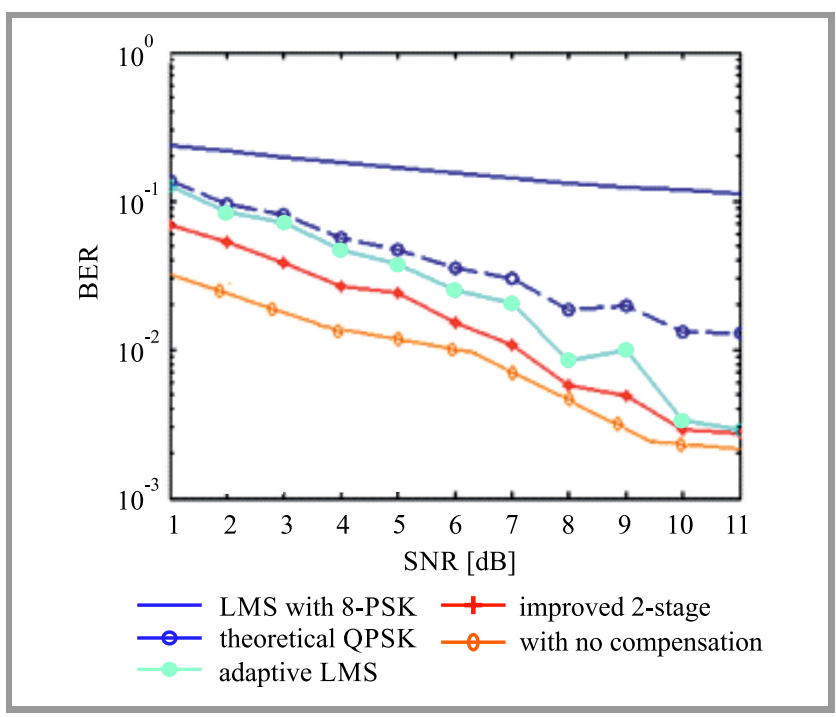

Fig. 9. Comparative analysis of BER between proposed algorithm and other algorithms.

QPSK and the improved two stage algorithm with 8-PSK is compared. The results show that the curve of adaptive LMS is distant from the theoretical curve of QPSK. Investigation reveals that the improved 2-stage algorithm with 8-PSK performs better than the adaptive LMS algorithm and is closer to the theoretical curve. The proposed algorithm relying on 8-PSK modulation outperforms the existing LMS algorithm using the QPSK modulation technique and significantly reduces BER by $8 \%$.

\section{Conclusion}

In this paper, phase error generated by phase noise in the CO-OFDM system is reduced. To accomplish this goal a phase noise estimation algorithm with 8-PSK modulation technique is proposed and implemented to reduce phase error. BER values of the adaptive LMS algorithm, improved 2-stage algorithm with QPSK and improved 2-stage algorithm with 8-PSK are compared. The improved 2-stage algorithm with 8-PSK significantly outperforms the adaptive LMS algorithm. This algorithm with 8-PSK reduces the bit error rate by $8 \%$ when compared with the existing adaptive LMS algorithm. Moreover, the problem of cycle slips is avoided using this algorithm. Investigations revealed that the proposed algorithm with the 8-PSK modulation technique is capable of providing a lower BER than 
the existing algorithms used for reducing phase noise in CO-OFDM systems.

\section{References}

[1] T. Rahman and C. Sacchi, "A cooperative radio resource management strategy for mobile multimedia LTE uplink", in Proc. IEEE Int. Conf. on Aerospace, Big Sky, MT, USA, 2014

(doi: 10.1109/AERO.2014.6836180).

[2] X. Zhang et al., "Power control algorithm in cognitive radio system based on modified shuffled frog leaping algorithm", AEU - Int. J. of Electron. and Commun., vol. 66, no. 6, pp. 448-454, 2012 (doi: 10.1016/j.aeue.2011.10.004).

[3] L. B. Le et al., "QoS-aware and energy-efficient resource management in OFDMA femtocells", IEEE Trans. on Wirel. Commun., vol. 12, no. 1 , pp. $180-194,2013$ (doi: 10.1109/TWC.2012.120412.120141)

[4] A. R. S. Bahai and B. R. Saltzberg, Multi-Carrier Digital Communications: Theory and Applications of OFDM. New York: Kluwer, 1999 (ISBN: 0-306-46296-6).

[5] P. Shelswell, "The COFDM modulation system: The heart of digital audio broadcasting", Electron. and Commun. Engin. J., vol. 7, no. 3, pp. 127-135, 1995 (doi: 10.1049/ecej:19950309).

[6] K. Sundaresan and S. Rangarajan, "Efficient resource management in OFDMA femto cells", in Proc. of the 10th ACM Int. Symp. on Mob. Ad Hoc Netw. and Comput. MobiHoc '09, New Orleans, LA, USA, 2009, pp. 33-42 (doi: 10.1145/1530748.1530754).

[7] P. H. Moos, "A technique for orthogonal frequency division multiplexing frequency offset correction", IEEE Trans. in Commun., vol. 42, no. 10, pp. 2908-2914, 1994 (doi: 10.1109/26.328961).

[8] A. G. Armada, "Understanding the effects of phase noise in orthogonal frequency division multiplexing (OFDM)", IEEE Trans. in Broadcast., vol. 47, no. 2, pp. 150-159, 2001 (doi: 10.1109/11.948268).

[9] L. Tomba and W. A. Krzymien, "Sensitivity of the MC-CDMA access scheme to carrier phase noise and frequency offset", IEEE Trans. in Veh. Technol., vol. 48, no. 5, pp. 1657-1665, 1999 (doi: 10.1109/25.790546).

[10] C. P. Lee, S. C. Lin, H. J. Su, and H. V. Poor, "Multiuser lattice coding for the multiple-access relay channel", IEEE Trans. on Wirel. Commun., vol. 3, no. 7, pp. 3539-3555, 2014 (doi: 10.1109/TWC.2014.2316496).

[11] A. Paurkabirian, M. D. T. Fooladi, and A. M. Rahmani, "Dynamic resource allocation for OFDMA femtocell networks: a game theoretic approach", J. of Telecommun. Syst., vol. 69, no. 1, pp. 51-59, 2018 (doi: 10.1007/s11235-018-0420-x).
[12] X. Yi, W. Shieh, and Y. Ma, "Phase noise effects on high spectral efficiency coherent optical OFDM transmission", J. of Light Wave Technol., vol. 26, no. 10, pp. 1309-1316, 2008 (doi: 10.1109/JLT.2008.919368).

[13] Y. Jianguo et al., "Comparison analysis on the performances of several synchronization algorithms for optical OFDM systems", Semiconductor Optoelectron., vol. 33, no. 4, pp. 544-547.

[14] A. J. Viterbi and A. M. Viterbi, "Non-linear estimation of PSKmodulated carrier phase with application to burst digital transmission", IEEE Trans. on Inform. Theory., vol. 29, no. 4, pp. 543-551, 1983 (doi: 10.1109/TIT.1983.1056713).

[15] A. Roberts et al., "Performance of dual-polarization OPSK for optical transport systems", J. of Light Wave Technol., vol. 27, no. 16, pp. 3546-3559, 2009 (doi: 10.1109/JLT.2009.2022484).

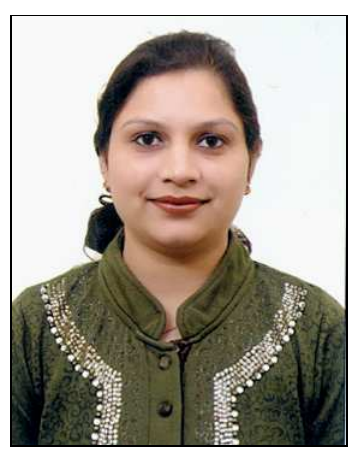

Manisha Bharti has completed her B.Tech. in Electronics and Communication Engineering (ECE) from Guru Nanak Dev University, Amritsar in 2002, M.Tech. in ECE from PTU, Jalandhar in 2007. She received her Ph.D. in 2017 from IKG PTU, Jalandhar in the field of Optical Communication and Networks. Her area of research includes optical communication and networks, analog, digital and broadband communication circuits, optical signal processing and VLSI. She has over 16 years of experience in Academics. She worked as Assistant Professor for over 7 years at AIACT\&R, Delhi, under Govt. of NCT of Delhi and is currently working as Assistant Professor in National Institute of Technology (NIT), Delhi.

Email: manishabharti@nitdelhi.ac.in

Department of Electronics and Communication

Engineering

National Institute of Technology (NIT)

Delhi, Sector A-7

Institutional Area, Narela

Delhi - 110040, India 